\title{
Identity and psychopathology:
}

\section{Bridging developmental and clinical research}

\author{
Margaux Verschueren ${ }^{1}$ \\ Laurence Claes ${ }^{1,2}$ \\ Amarendra Gandhi ${ }^{1}$ \\ Koen Luyckx ${ }^{1,3}$ \\ ${ }^{1}$ University of Leuven, Leuven, Belgium \\ ${ }^{2}$ Antwerp University, Antwerp, Belgium \\ ${ }^{3}$ UNIBS, University of the Free State, Bloemfontein, South Africa
}

(The present paper is based on the keynote speech by K. Luyckx held at the conference:

"Moving the identity field forward: Bridging developmental and clinical research") 


\begin{abstract}
Research has demonstrated the important impact of identity on psychosocial functioning in both community and clinical populations. The present paper aims to identify different mechanisms through which identity may be related to psychopathology. Emphasis is placed on neoEriksonian identity models targeting identity mechanisms both at the structural and process levels. With respect to psychopathology, the present paper focuses mainly on disturbed eating behaviors and non-suicidal self-injury, with both behaviors sharing a focus on the body. Emerging research lines are identified and recent research is discussed as a sample case of how developmental theorizing on identity can yield insights in the emergence and development of psychopathological behaviors. In integrating these research lines, the present article discusses emerging themes originating from the field and provides important avenues for future research and intervention efforts.
\end{abstract}


Identity formation is commonly framed as the central developmental task of adolescence and the transition into adulthood, extending from the early teens well into the twenties (Arnett, 2000; Erikson, 1968). This process of identity formation essentially addresses the question "Who am I and where am I heading in life?” (Schwartz, Luyckx, \& Vignoles, 2011). Coming up with an answer to this life-defining question not only becomes important throughout the second and third decades of life but remains so throughout the entire life-span (Erikson, 1950; Kroger, 2000). A vast amount of research has demonstrated that how one addresses these identity issues is not only related to adaptation and adjustment, but also to suboptimal functioning, distress, and even psychopathology (e.g., Crocetti, Luyckx, Scrignaro, \& Sica, 2011; Luyckx et al., 2008; Schwartz, Beyers, et al., 2011; Wiley \& Berman, 2013). These latter findings should not come as a surprise as identity research and theorizing partially emerged from clinical observations, as can be derived from Erikson's $(1950,1968)$ writings. However, for identity research to reach its full potential in terms of informing clinical practice and (preventive) interventions, we still have a long way to go. A main reason for this rather unfortunate state of the field is the considerable gap between developmental and clinical literatures (Kaufman, Montgomery, \& Crowell, 2014; Pasupathi, 2014). In the present paper, and inspired by a developmental psychopathology framework (Kaufman et al., 2014), we try to bridge these two bodies of literature by demonstrating the added value of neo-Eriksonian identity models for our understanding of pathological behaviors in community and clinical samples.

\section{Assessing Identity Structure and Processes}

Our main inspirations for conceptualizing and measuring identity were Erikson's (1968) identity theorizing and Marcia's (1966) identity status paradigm. Partially inspired by Marcia's (2006) seminal article on the link between identity and personality disorders, we commonly assess identity at different levels - covering identity structure as well as behavioral identity 
processes. As conceptualized by Erikson $(1950,1968)$, one's identity structure is characterized by a certain degree of synthesis and confusion, and successful identity development is commonly portrayed as the extent to which synthesis predominates over confusion. Individuals scoring high on identity synthesis experience self-continuity over time and across situations, and have developed a stable set of goals, plans, and beliefs (Erikson, 1968; Kaufman, Montgomery, \& Crowell, 2014). Individuals high on identity confusion experience a fragmented sense of self and limited self-directed decision making (Schwartz, Zamboanga, Luyckx, Meca, \& Ritchie, 2013). They struggle to find purpose and direction in their life (Schwartz, Zamboanga, Wang, \& Olthuis, 2009).

Marcia (1966) developed the status paradigm and defined two key identity processes as behavioral indicators of this identity structure: exploration, or experimenting with identity alternatives; and commitment, or adhering to a set of identity-defining goals and values. Based on these two processes, Marcia derived four statuses: achievement (individuals with strong commitments following a period of exploration), foreclosure (individuals having enacted commitments without previous exploration), moratorium (individuals who are still exploring and have not enacted strong commitments yet), and diffusion (individuals who are not proactively exploring and do not have strong guiding commitments).

Several researchers have proposed refined models in which exploration and commitment have been unpacked into a larger set of processes. In one such model, Luyckx, Goossens, and Soenens (2006) distinguished four processes subsumed under two complementary cycles. The first cycle (identity formation) builds on Marcia's (1966) work and captures the processes through which individuals explore alternatives and make commitments: exploration in breadth, or the pro-active exploration of alternatives, and commitment making, or the adherence to a set of choices. The second identity cycle (identity evaluation) captures the processes through which individuals (re-)evaluate their identity choices. This second cycle is 
also described in terms of two processes: exploration in depth, or the evaluation of current commitments, and identification with commitment, or the degree to which these commitments become integrated to the individual's sense of self.

To illustrate these two identity cycles, we refer to the higher education context, in which adolescents and young adults consider pursuing a college degree. First, they may gather information about different college majors such as physical therapy, psychology, and biology (exploration in breadth), followed by making a clear choice by enrolling in a psychology program (commitment making). Subsequently, once the academic year has started, they may evaluate the degree to which the courses correspond with their internal standards (exploration in depth) and, as a consequence, identify with or feel certain (or not) about becoming a psychologist (identification with commitment). However, due to a variety of contextual and/or intrapersonal factors, individuals may become stuck in their identity search and experience difficulties enacting commitments (Schwartz, Côté, \& Arnett, 2005). The indecisive student may keep on hesitating about the psychology program, without actively looking into other options, which would result in feelings of uncertainty and incompetence. Consequently, ruminative exploration was added as a fifth process that hinders identity development. As individuals scoring high on ruminative exploration are partially troubled by what they perceive as inadequate progress towards personally important goals, they experience difficulty settling on satisfying choices and keep asking themselves the same questions (Luyckx et al., 2008).

The model proposed by Luyckx et al. (2008) also allows for a fine-grained analysis of identity statuses. Through the use of cluster analysis on identity process scores, several identity clusters emerged, of which some were similar to Marcia's original statuses (e.g., Crocetti et al., 2011; Luyckx et al., 2008; Schwartz, Beyers, et al., 2011). Both achievement and foreclosure were characterized by high scores on commitment processes and low scores on ruminative exploration. In addition, achievement was characterized by substantially higher scores on 
exploration in breadth and exploration in depth. Hence, individuals who explore when making commitments may be most likely to experience identity synthesis and to ascribe meaning to their lives. Individuals in foreclosure, however, may be less likely to experience such a sense of personal meaning (Schwartz, Beyers, et al., 2011; Waterman, 2007).

As moratorium is associated with distress and low self-worth, several authors have questioned whether this status is truly an adaptive step toward identity synthesis. To the extent that young people are engaged in a "perpetual moratorium", they may experience aggravated identity confusion as well (Côté \& Schwartz, 2002; Marcia, 2002). Luyckx et al. (2008) indeed found that individuals in moratorium scored high on ruminative exploration in addition to proactive exploration. An extended moratorium phase may denote a type of arrested development, blocking individuals from forming commitments and progressing to achievement (Côté \& Levine, 2002). Further, previous research has identified individuals in "searching moratorium", who score high on all exploration processes, but still maintain a set of commitments (Mannerström, Hautamäki, \& Leikas, 2017; Schwartz, Beyers, et al., 2011). These individuals could thus be willing to change existing commitments, while exploring new alternatives. With respect to diffusion, Luyckx et al. (2008) distinguished troubled from carefree diffusion (Archer \& Waterman, 1990; Marcia, 1989). Both clusters were characterized by low scores on commitment processes and limited exploration in breadth and in depth. However, more so than carefree-diffused individuals, troubled-diffused individuals attempted to explore identity issues. However, they did so in a predominantly ruminative manner. Thus, whereas troubleddiffused individuals might be unable to take proactive steps in identity development, carefreediffused individuals seem to be uninterested or unmotivated to do so (Luyckx, Klimstra, Schwartz, \& Duriez, 2013; Schwartz, Beyers, et al., 2011). Finally, an undifferentiated cluster emerged (Luyckx et al., 2008; Schwartz, Beyers, et al., 2011), consisting of individuals who scored close to the sample means on all identity processes. 


\section{The Promise of Identity Research for Understanding Psychopathology}

Both identity structure and processes have repeatedly been related to indicators of adjustment and distress in community research (e.g., Crocetti et al., 2011; Luyckx et al., 2008; Schwartz, Beyers, et al., 2011; Wiley \& Berman, 2013). Recently, there has been increasing research and theorizing focusing on the link between identity and psychopathology: do certain identity processes and mechanisms render individuals vulnerable for displaying psychopathology? Or, conversely, can one's identity also protect against experiencing pathological symptoms and behaviors? Both a view on one's degree of identity synthesis/confusion and a fine-grained perspective on identity commitment and exploration processes (and how they combine into certain identity clusters or statuses) have great potential to improve our understanding of how normative identity development may contribute to, and may become intertwined with, psychopathology. Further, inspired by developmental psychopathology (Kaufman et al., 2014), an explicit focus on normative-developmental constructs can inform us as to how, and under which circumstances, identity development may go awry and give rise to clinical identity problems or disorders. Hence, such a continuum perspective on identity may provide important insights for our understanding of the identity psychopathology link.

Such an integrative perspective on identity in which both normative and dysfunctional mechanisms are mutually informative for understanding psychopathology emerges prominently in an alternative model for personality disorders as forwarded in DSM-5 Section III (American Psychiatric Association [APA], 2013). In this alternative model, identity is recognized as a potential factor in all personality disorders [and not just Borderline Personality Disorder as was the case in DSM-IV-R (APA; 2000) and earlier editions]. More specifically, this alternative model describes personality pathology in terms of impairment in personality functioning (Criterion A) and having one or more pathological personality traits (Criterion B). Moderate or 
greater impairment in personality functioning is manifested by characteristic difficulties in two or more of the following four domains: identity, self-direction, empathy, and intimacy. When looking at how these domains of identity and self-direction are defined, some references to (neo-)Eriksonian notions of identity structure and decision-making in terms of exploring and committing to life goals can be found. However, the many nuances of key identity constructs forwarded in developmental psychology are not included (Pasupathi, 2014), necessitating integrating more developmental research into the identity-psychopathology linkages.

Such a call for a more fine-grained developmental study of identity in understanding psychopathology has been advanced by several authors. Recently, Klimstra and Denissen (2017) have proposed an integrative framework in which aspects of psychological functioning and identity notions of structure and process are incorporated in a multidimensional space. More specifically, identity components would be positioned in the center of this multidimensional space and aspects of psychological functioning could be closer or further away from the center. The distance between both would then represent the degree of relevance of these aspects to one's identity. Furthermore, Klimstra and Denissen (2017) argue that one's identity and psychopathological symptoms may become increasingly interwoven over time. Furthermore, Klimstra and Denissen argue that one's identity and psychopathological symptoms may become increasingly interwoven over time. In 2014, Kaufman, Montgomery, and Crowell explicitly advocated for a more integrative study of clinical and developmental perspectives in order to advance our understanding of identity problems and dysfunction. The need for such an integrative study has been emphasized again in their more recent paper in which they have advanced an explanatory framework of identity pathology drawing from complementary evidence from social, developmental, behavioral, clinical, and neuroscience literatures (Kaufman \& Crowell, 2018). Further, Kaufman et al. (2014) advocate for a clear definition of different identity terms as both developmental and clinical literatures would greatly benefit 
from a shared lexicon in which key constructs are defined unambiguously. To build bridges between both literatures, the core building blocks need to be clearly delineated and agreed upon such that definitional confusion can be limited. For instance, although terms such as identity disturbance and diffusion are often used interchangeably, it remains to be determined when and how a normative sense of identity diffusion as defined in neo-Eriksonian traditions sets the stage for or transforms into clinical identity disturbance (Kaufman et al., 2014; Kernberg, 1984; Schmeck, Schlüter-Müller, Foelsch, \& Doering, 2013) and, consequently, may render individuals especially vulnerable for experiencing psychopathology (Westen, Betan, \& DeFife, 2011).

In the present paper, we do not claim (or even aim at) resolving these difficult issues. However, we aim at initiating some first steps in this direction by demonstrating how developmental notions of identity structure and process can inform our understanding of certain psychopathological behaviors. In doing so, we mainly seek to describe research examining behaviors such as eating disorder (ED) symptomatology and non-suicidal self-injury (NSSI), as both share a common ground in that they are seen as behaviors that "target the body". The body has long been described as crucial for identity development, although integrative developmental research focusing on body-identity linkages is somewhat lagging behind (Erikson, 1968; Kling, Wängqvist, \& Frisén, 2018; Orbach \& Mikulincer, 1998; Wängqvist \& Frisén, 2013). The feeling of being at home in one's body is indeed a key component of the self (Erikson, 1968), with the bodily self developing in close interaction with one's general sense of self (Cash \& Deagle, 1997; Orbach \& Mikulincer, 1998). Along these lines, and as further detailed below, body image problems may not only affect how individuals think about themselves (Wängqvist \& Frisén, 2013), but these body image issues may become closely intertwined with a sense of identity diffusion or even clinical identity disturbance. Such a suboptimal self-trajectory, in turn, may make individuals especially vulnerable for body 
dissatisfaction, and partially as a consequence, pathological self-harm behaviors such as disordered eating and NSSI (Muehlenkamp \& Brausch, 2012; Orbach, 1996; Vartanian, Hayward, Smyth, Paxton, \& Touyz, 2018). Below we describe recent research efforts examining how identity issues may become intertwined with such self-harm behaviors.

\section{Identity and ED Symptoms and Pathology}

EDs are prevalent in adolescents and emerging adults and have a devastating impact on both physical and mental health. The DSM-5 (APA, 2013) describes anorexia nervosa (AN), bulimia nervosa $(\mathrm{BN})$, and binge-eating disorder (BED) as the three main EDs. AN is characterized by a restriction of energy intake relative to energy requirements, which leads to a significantly low body weight. Additionally, an intense fear of gaining weight and disturbed body- and weight experiences are emphasized. BN is characterized by recurrent binge-eating episodes combined with inappropriate compensatory behavior to prevent weight gain (purging, laxative misuse, fasting, etc.). Individuals with $\mathrm{BN}$ also emphasize their body shape and weight disproportionately in their self-evaluation. Finally, BED is characterized by recurrent bingeeating episodes without recurrent inappropriate compensatory behavior to prevent weight gain.

Overall, EDs have a peak onset between the ages of 15 and 19 years (Micali, Hagberg, Petersen, \& Treasure, 2013). However, across development, patients may experience diagnostic shifts, with a typical shift from more restrictive ED diagnoses to more binge-eating/purging types over time (Fairburn, Cooper, \& Shafran, 2003; Vandereycken \& Noordenbos, 2008). In general, BN and BED seem to be more prevalent than AN in both adolescents and adults (SEN; 1998; Swanson, Crow, Le Grange, Swendsen, \& Merikangas, 2011). Along with these formal diagnoses, disturbed eating behaviors, referring to unhealthy weight control behaviors that do not fulfill diagnostic criteria of an ED (e.g., fasting, taking diet pills or laxatives, vomiting, binge-eating), are highly prevalent in adolescents (approximately 57\% for girls and 30\% for boys; Croll, Neumark-Sztainer, Story, \& Ireland, 2002) and emerging adults (approximately 
25\% for women and men; Quick \& Byrd-Bredbenner, 2013). Generally, females report more disturbed eating behaviors and ED cognitions than males (Lewinsohn, Seeley, Moerk, \& Striegel-Moore, 2002; Micali, Ploubidis, De Stavola, Simonoff, \& Treasure, 2014).

As the age of onset indicates, adolescence and emerging adulthood represent crucial life periods in which individuals may become vulnerable to display eating symptoms or even an ED. These life periods are characterized by various bodily changes and a great concern with social acceptance (Elkind \& Bowen, 1979; Harter, 1999), which may make adolescents especially vulnerable to internalizing certain beauty standards that idealize slim and fit bodies (Suisman et al., 2014). Moreover, research increasingly indicates that the process of identity formation is closely related to the development of such disturbed eating symptoms. Verstuyf, Van Petegem, Vansteenkiste, Soenens, and Boone (2014) found that individuals' identity styles (Berzonsky, 2004) are differentially related to the internalization of the body perfect ideal and unhealthy dietary restrictions. More specifically, individuals who maintain active and critical attitudes towards identity-relevant information (i.e., information-oriented style) have been found to distance themselves from the body perfect ideal, whereas individuals who are vulnerable to socially expected goals and values (i.e., normative style) tend to internalize the body perfect ideal and adopt appearance-focused and unhealthy dietary restrictions. Finally, individuals who tend to avoid making identity-related decisions (i.e., diffuse-avoidant style) seem to be vulnerable for adopting unhealthy eating patterns.

The identity-ED link also seems to be important in clinical samples, as patients with an ED have been found to experience more identity confusion than individuals without this diagnosis (Auslander \& Dunham, 1996; Schupak-Neuberg \& Nemeroff, 1993; Sparks, 1993). Focusing on identity processes and statuses, we (Verschueren et al., 2017) compared a clinical ED sample to an age-matched community sample and found that patients with an ED experienced far greater difficulties in forming a personal identity than community individuals 
did. More specifically, ED patients scored significantly lower on most adaptive identity processes (i.e., commitment making, identification with commitment, and exploration in breadth) and significantly higher on ruminative exploration as compared to community controls. The differences in the commitment processes and ruminative exploration were accompanied by especially large effect sizes. Hence, it seems particularly difficult for patients with an ED to make self-endorsed identity commitments, possibly due to a ruminative identity process. Interestingly, when examining the identity statuses that emerged in the combined sample of patients with an ED and community controls, an identity disorder status was identified, which consisted solely of patients with an ED. This identity status captured individuals scoring very low on all adaptive identity processes combined with a very high score on ruminative exploration (resembling a more pathological form of troubled diffusion) and, hence, indicated serious identity problems or even identity disturbance. Altogether, the results of this study suggested that patients with an ED experience more trouble in making identityrelated choices and pro-actively exploring identity issues, and that some patients seem to experience an identity disorder that is associated with maladaptive functioning.

Although an increasing body of research has examined the relation between identity and eating symptomatology, no consensus exists on how the directionality of these associations can be understood. In this respect, most theorists agree that identity difficulties are already present before the onset of ED symptomatology. Bruch $(1981,1982)$, described AN as developing due to impairments in overall identity development. A disrupted identity may motivate adolescents to search for a (maladaptive) source of self-definition, which is often found in the controllable and culturally valued aspect of body weight (Bruch, 1981; Polivy \& Herman, 2007; SchupakNeuberg \& Nemeroff, 1993). Vartanian et al. (2018) indeed found that individuals with lower self-concept clarity (somewhat resembling Erikson's identity synthesis; Campbell, Trapnell, Heine, Katz, Lavallee, \& Lehman, 1996) seem to be especially at risk for internalizing Western 
beauty ideals and for comparing their appearance more frequently to that of others. In turn, internalization of beauty ideals and appearance comparison may be associated with body image concerns and disordered eating. Hence, restrictive eating may represent a (subconscious) quest for a stronger sense of self and individuation (Bruch, 1981; Casper, 1983).

Building further on these ideas, an increasing number of theorists state that eating symptomatology may not only arise from identity problems, but that it could also play into one's identity development and even derail normal identity development. Corning and Heibel (2016) note that most individuals with disturbed eating behaviors are preoccupied with their body weight and shape, with body weight representing a disproportionate share of their identity. Fairburn et al. (2003) describe a dysfunctional system for self-evaluation as one of the core deficits of patients with an ED. This system would lead patients with an ED to evaluate themselves almost entirely in terms of their eating habits and body shape, while alternative sources of self-worth are lacking. Hence, when these self-aspects are under attack (e.g., by weight teasing or social comparison), they may be experienced as direct threats to the self (Corning \& Heibel, 2016; Polivy \& Herman, 2007) that, in turn, motivate patients to adhere even more to strict dieting rules. Indeed, Schupak-Neuberg and Nemeroff (1993) described how controlling food intake could represent a regulation of one's identity in patients with an ED. This food-self metaphor would not only explain why restrictive eating brings inner calmness to the patient, but could also offer insights into binge-purging behavior. Polivy, Herman, and McFarlane (1994) discussed that, when ED patients are confronted with a threat to their selfimage, anxious and nervous feelings emerge, which in turn drive them toward binge eating as well. During a binge, the collapse of bodily regulation may represent a collapse of one's selfdefinition as well (Schupak-Neuberg \& Nemeroff, 1993). The cognitive narrowing that occurs during the binge blocks self-awareness for a short period of time (Heatherton \& Baumeister, 1991). After the binge, when self-awareness returns, the remaining negative feelings are then 
often expelled by purging (Schupak-Neuberg \& Nemeroff, 1993). Several authors have described similar ideas regarding the functional mechanisms of binge-purging behavior, in which this type of behavior may help individuals to avoid dealing with identity issues or escape from thoughts about the self (Heatherton \& Baumeister, 1991; Herman \& Polivy, 1988; Wheeler, Adams, \& Keating, 2001).

Altogether, identity functioning and eating symptomatology seem to be closely and reciprocally related to one another, as visually represented in Figure 1. Briefly summarized, individuals experiencing more identity diffusion may also be more susceptible to internalize the body perfect ideal - promoting a slender or muscular body for women and men, respectively. Consequently, this internalization may fuel individuals to consider this perfect body ideal as a source of self-definition. This can, in turn, stimulate the wish to be thinner and trigger disturbed eating behavior, especially in women. Once individuals have engaged in these types of behavior, it may install a harmful cycle in which the body and eating regulation become an increasingly central part of one's identity and self-esteem. Hence, gaining weight may be regarded as a threat to one's identity, which contributes to the development of a vulnerable sense of self and, consequently, identity diffusion.

To examine such a bidirectional relationship, we conducted a two year, three-wave longitudinal study with a community sample of adolescents and emerging adults (Verschueren et al., 2018). Cross-lagged analyses indicated that identity confusion appeared to increase one's vulnerability to experience body dissatisfaction and display bulimia symptoms over time relative to the rest of the sample, whereas identity synthesis seemed to protect against the emergence of both. Additionally, identity synthesis seemed to protect against the development of drive for thinness as well. Hence, these findings seem to be in line with the idea that adolescents with certain doubts about their identity may be more vulnerable to adopting the body perfect ideal of Western society and experience more eating symptomatology (Berzonsky, 
2004; Verstuyf et al., 2014). At the same time, our findings indicated that body dissatisfaction and bulimia symptoms positively predicted identity confusion and negatively predicted identity synthesis over time as well. Eating symptomatology thus seems to hamper normative identity development over time, as individuals with disturbed eating behavior may experience a narrowly defined identity characterized by an overvaluation of body size and weight (Corning \& Heibel, 2016; Fairburn et al., 2003).

In line with these results, Corning and Heibel (2016) have advocated for the promotion of a healthy identity development in ED prevention programs, in which adolescents learn to bolster other self-aspects besides their body image and appearance. Such an approach may result in individuals being able to develop a more well-rounded identity and self-esteem in which other self-aspects are valued, and may allow them to experience more optimal selfdevelopment over time. Similarly, in ED intervention programs, it seems crucial for therapists to pay special attention to identity formation by offering patients tools in which they can learn to break the vicious cycle linking identity problems with eating symptomatology.

\section{Identity and NSSI}

NSSI is defined as the repetitive, direct, and deliberate destruction of one's body tissue without an intention to die, and for purposes not socially sanctioned (Nock \& Favazza, 2009). NSSI represents a distinct category of behavior that falls under the umbrella of self-directed violent behaviors and has been increasingly identified as an important public health concern, especially among adolescents. In the past, NSSI has been described using various terms such as self-mutilation, partial or para-suicide, auto-mutilation, delicate cutting, deliberate self-harm, and self-injurious behaviors. Common examples of NSSI include self-cutting, self-scratching, head banging, self-hitting, and self-burning. As NSSI involves direct destruction of the body tissue, indirect self-harming behaviors such as alcoholism, self-poisoning, substance abuse, and EDs are not considered as NSSI. 
NSSI is related to both distal and proximal intrapersonal (e.g., personality) and interpersonal (e.g., attachment) vulnerability factors (Nock, 2009) and negative mental health outcomes (e.g., depressive and anxiety symptoms; Kerr, Muehlenkamp, \& Turner, 2010). Additionally, approximately 50-75\% of individuals with a history of NSSI attempt suicide at some point in their life (Nock, Joiner, Gordon, Lloyd-Richardson, \& Prinstein, 2006). These substantial negative mental health outcomes of NSSI underscore the need for a better understanding of NSSI (Mullins-Sweatt, Lengel, \& Grant, 2013). NSSI has been added to the “Conditions for Further Study" section in the DSM-5 (APA, 2013) to stimulate further research on this set of destructive behaviors. Currently, the criteria for NSSI disorder include: (A) engaging in NSSI on 5 or more days in the last year, (B) engaging in NSSI to relieve negative feelings, interpersonal difficulty or to induce positive feelings, (C) NSSI being associated with interpersonal difficulties, preoccupation, and thinking about NSSI even when not acted upon, (D) the behavior is not socially sanctioned, (E) causes distress or interference in functioning, and (F) does not occur exclusively during psychotic episodes, delirium, and substance intoxication or withdrawal (APA, 2013, p. 803).

In community samples, lifetime prevalence rates of NSSI are estimated to be around 17.2\% among adolescents, $13.4 \%$ among young adults, and 5.5\% among adults (Muehlenkamp, Claes, Havertape, \& Plener, 2012; Swannell, Martin, Page, Hasking, \& St John, 2014). In clinical samples, NSSI rates are even higher (Briere \& Gil, 1998) and have been found to range from $23.8 \%$ to $31.0 \%$ in patients with an ED (Cucchi et al., 2016; Muehlenkamp, Claes, Smits, Peat, \& Vandereycken, 2011) and rise up to $70-75 \%$ in patients with Borderline Personality Disorder (Claes, Van den Eynde, Guillaume, Vogels, \& Audenaert, 2012; Kerr et al., 2010). A recent meta-analysis by Bresin and Schoenleber (2015) has demonstrated that NSSI is slightly more common in women than in men. This gender difference is more pronounced in clinical samples than in community samples. Gender differences also exist in the type of methods 
endorsed by individuals to engage in NSSI - women tend to engage more often in cutting behaviors, whereas men are more likely to engage in self-battery (e.g., self-hitting, head banging; Bresin \& Schoenleber, 2015). Irrespective of the type of sample (community or clinical sample), the age of onset of NSSI peaks around the age of 14-15 years. A second peak is observed around the ages of 20 and 24 years in community and clinical samples, respectively (Gandhi, Luyckx, Baetens, et al., 2018; Plener, Schumacher, Munz, \& Groschwitz, 2015). As in the case of EDs, the higher prevalence and greater likelihood of NSSI onset in adolescence and emerging adulthood again raises the possibility of the role of identity development in increasing one's vulnerability to engage in NSSI.

The association between NSSI and identity formation has long been suggested in extant literature. For example, Podvoll (1969) observed that patients engaging in self-cutting or related self-harm behaviors transformed their symptoms into an identity (slashers, cutters, scratchers, etc.). Based on the observation of patients admitted to a psychiatric hospital, Podvoll reported that patients with NSSI have an enduring feeling of emptiness and a disbelief in their own growth potential. They often believe that the NSSI is all they possess and that nothing else can substantially define their core sense of self (Podvoll, 1969). Consequently, stopping such selfharm behaviors may be experienced to lead to inner emptiness and severe identity diffusion.

Podvoll's initial observations have been echoed by a number of researchers. Asch (1971), for instance, stated that NSSI may serve as a way of counteracting a sense of emptiness and identity confusion. Interestingly, more recent qualitative studies have drawn similar conclusions. Using thematic analysis of online autobiographical accounts of NSSI on blog posts, Breen, Lewis, and Sutherland (2013) concluded that NSSI may serve as a means of developing a sense of identity ("I am a self-injurer"), through connecting with others who engage in similar behaviors. Hence, despite the problematic and self-destructive nature of NSSI, it may provide individuals with a subjectively experienced coherent sense of self as it may serve 
as a springboard to connect with a social group (i.e., the group of self-injurers). Subsequently, individuals may draw from such a collective identity to derive a personal sense of self that can persist across time (cf. Klimstra \& Denissen, 2017).

A recent study by Claes, Luyckx, and Bijttebier (2014) found that NSSI was negatively associated with identity synthesis and positively associated with identity confusion in a sample of high school students. Further, even when controlling for depressive symptoms, age, and gender, they found that identity confusion explained additional variance in NSSI. These findings have been replicated in multiple cross-sectional studies in both community and clinical samples (Claes et al., 2015; Gandhi, Luyckx, Goossens, Maitra, \& Claes, 2016; Luyckx, Gandhi, Bijttebier, \& Claes, 2015a, 2015b). Relatedly, the link between experienced identity distress in specific life domains (long-term goals, career choice, friendships, sexual orientation and behavior, religion, values and beliefs, and group loyalties; Berman, Montgomery, \& Kurtines, 2004) and NSSI has been explored as well. Gandhi, Luyckx, Maitra, and Claes (2015) demonstrated that the subjective impairment accompanying such identity distress predicted the lifetime prevalence of NSSI even when controlling for age, gender, anxiety, and depression.

Although such cross-sectional studies appear to suggest that disturbances in identity formation can increase vulnerability to NSSI, a longitudinal study in high school students by Gandhi, Luyckx, Maitra, Kiekens, Verschueren, and Claes (2017) provides a more nuanced picture of the identity-NSSI link. Cross-lagged analyses suggested the presence of a bidirectional association between NSSI and identity synthesis/confusion, in much the same way as identity and ED symptoms were related (Verschueren et al., 2018). That is, individuals experiencing less identity synthesis and increased identity confusion were more likely to engage in NSSI one year later relative to the rest of the sample. Furthermore, engagement in NSSI at Time 1 was also found to be associated with further relative decreases in identity synthesis and increases in identity confusion. The potential existence of such reciprocal associations was also 
found in a cross-sectional study linking identity statuses to both past and current NSSI (Luyckx et al., 2015b). Luyckx and colleagues reported that individuals in the moratorium status were most likely, and individuals in the achievement status were least likely, to report NSSI in the past. This finding indicates that engaging in NSSI earlier on in the lifespan may delay identity formation. On the other hand, troubled-diffused individuals were most likely to report current (but not past) NSSI, suggesting that identity diffusion might play into NSSI as well. That is, NSSI could constitute a problematic coping strategy for dealing with identity issues. In line with Gandhi et al. (2017), these results do indicate that the association between identity and NSSI may be reciprocal.

Based on the existing theoretical understanding of NSSI and identity formation and the findings of the aforementioned cross-sectional and longitudinal research, we propose the following model (Gandhi et al., 2017; see also Figure 2) to explain the observed bidirectional relation between identity formation and NSSI. As detailed below, common pathways linking Figures 1 and 2 can be identified. Engaging in NSSI may help individuals who experience identity confusion in at least two ways. First, it can help them in managing negative affect. An increase in identity confusion has been shown to be associated with a corresponding increase in distress and internalizing symptoms (Luyckx, Klimstra, Duriez, Van Petegem, \& Beyers, 2013). To regulate this negative affect, adolescents experiencing identity diffusion may engage in NSSI. Second, engaging in NSSI may offer them an alternate sense of self. NSSI may represent a disproportionate aspect of one's identity and may even prompt some individuals to form the negative identity of a self-injurer. Negative identities, according to Erikson (1968), are those that go against the values and attitudes prevalent in society. Indeed, apart from the group that encourages NSSI, the negative identity of the self-injurer is less likely to receive validation from one's immediate interpersonal context. As identities require "inner assuredness of anticipated recognition from those who count" (Erikson, 1968, p. 165) for their stability, the 
identity of a self-injurer may remain short-lived (Côté \& Levine, 1987). Destabilization of the self-injurer identity may lead individuals back to an increased sense of identity diffusion.

\section{Common Pathways: The Potential Trans-Diagnostic Value of Identity}

Based on the research lines described above, common pathways linking identity to certain body-related psychopathological behaviors may be identified. Not surprisingly, ED and NSSI behaviors are highly comorbid (Claes, Klonsky, Muehlenkamp, Kuppens, \& Vandereycken, 2010; Svirko \& Hawton, 2007) and share various risk factors (such as emotion dysregulation, dissociation, low body regard, and self-objectification; Claes \& Muehlenkamp, 2014). Importantly, both behaviors are described as disturbances in the way the individual experiences one's own body (Orbach, 1996; Stanghellini, Castellini, Brogna, Faravelli, \& Ricca, 2012). Individuals engaging in ED/NSSI behavior have been found to generally experience their body as a physical object that can be looked at by others, in which a firstperson perspective is lacking. This self-objectification and materialization of the body results in "a lived body for others" that can be judged or criticized (Stanghellini et al., 2012). Hence, being disconnected from internal bodily experiences, the individual is especially vulnerable to engage in self-harm and/or disturbed eating (Muehlenkamp \& Saris-Baglama, 2002; Muehlenkamp, Swanson, \& Brausch, 2005). This common risk factor does not only add to the high comorbidity of ED and NSSI, but may also relate to their interplay with identity. Research has indicated that, when experiencing this "lived body for others", the gaze of others on the body becomes highly salient to one's identity as well (Stanghellini et al., 2012). In these individuals, one's identity will be almost entirely reduced to the external appearance of the body and individuals will define themselves through evaluations of the other (Stanghellini et al., 2012), similar to the aforementioned theories of Corning and Heibel (2016) and Fairburn et al. (2003) in indivdiuals with an ED. The common mechanisms with identity described above are believed to be present in community samples presenting with subclinical pathological behaviors 
as well as in clinical samples, where the pathways between identity and body-related pathology would be stronger and could possibly represent a pathological dynamic.

To summarize, identity confusion can bring great distress and feelings of emptiness, which may stimulate individuals to search for a new source of self-definition, be it adaptive or maladaptive (Bruch, 1981; Campbell, 1990). As discussed above, the body represents an important component of the self and is highly valued in Western societies. When confronted with identity confusion and in an attempt to restore their identity, the body may offer some individuals a (maladaptive) source of self-definition. Hence, both disturbed eating and NSSI may be symptomatic of a rather maladaptive quest to experience a coherent sense of self, with the body being perceived as an object that can be controlled. As both behaviors may represent a primary source of self-esteem and identity for these individuals lacking a core sense of self, such an overemphasis and objectification of the body can be interpreted as a pseudo-identity that can only offer temporary security. As alternative sources of self-esteem and identity are lacking, when confronted with an identity threat, these individuals will lack sources of selfworth (such as occupational or friendship domains) on which to rely. Moreover, as this "ED/NSSI identity" is not socially validated and only provides temporary security, it is highly vulnerable and often yields feelings of identity diffusion. Finally, to cope with feelings of insecurity and self-doubt, both NSSI and ED symptomatology have emotion regulatory capacities that are often used when faced with a challenge. As identity diffused individuals often experience distress and are prone to internalizing symptoms, self-harming or disturbed eating may help them narrow their cognitive attention on the body. In this way, they avoid dealing with identity issues, which keeps the pathological cycle going.

Based on these common hypothesized pathways of identity with ED and NSSI, future research should address whether similar mechanisms would be at work in other pathologies as well, as identity problems have been described as a trans-diagnostic marker for 
psychopathology (Kaufman et al., 2014). Somewhat similar to the earlier discussed alternative model for personality disorders in DSM-5, the National Institute for Mental Health (NIMH) has recently described "Perception and Understanding of Self" as a dimensional construct in their Research Domain Criteria (RDoC). The RDoC represents a research framework for investigating and classifying mental disorders, based on dimensions of observable behavior and neurobiological measures. It aims to understand the nature of mental health, but does not provide a diagnostic guide. The "Perception and Understanding of Self" comprises processes involved in agency and self-knowledge and promotes self-awareness, self-monitoring, and selfknowledge. Agency is described as recognizing oneself as the agent of one's actions and thoughts, whereas self-knowledge comprises the ability to make judgements about internal states and traits. Both constructs share a common ground with the identity processes of exploration and commitment focusing on pro-actively engaging in the identity quest (Luyckx et al., 2008), and the identity synthesis construct of Erikson (1968), in which one experiences oneself as internally consistent. The RDoC framework describes this "Perception and Understanding of Self'-dimension as an important element in trying to understand the development of mental health problems, as it comprises important abilities that can protect or harm an individual vis-à-vis various mental issues.

In line with these recent developments, an increasing number of studies describe identity formation as playing an important role in non-body related disorders as well (such as hoarding and compulsive buying; Claes, Luyckx, Vogel, Verschueren, \& Müller, 2018; Claes, Müller, \& Luyckx, 2016; Dittmar, Long, \& Bond, 2007; Reeves, Baker, \& Truluck, 2012). Hence, similar pathways identified for the ED/NSSI-identity interplay may apply to these disorders as well. With regard to compulsive buying, Reeves et al. (2012) have described how the acquisition of nonessential goods may offer fulfillment for individuals with an "empty self" (Cushman, 1990). Doing so, buying material goods would offer a way to restore the "empty self" and would 
complete one's identity. Similarly, Dittmar et al. (2007) underscored how material goods may serve as potential identity substitutes, in which they become an expression of "who one is" (Wicklund \& Gollwitzer, 1982). These theoretical ideas offer an understanding of compulsive hoarding as well, as it explains why it is difficult for these individuals to get rid of their possessions - which would feel like losing a part of one's identity (Frost, Kyrios, McCarthy, \& Matthews, 2007).

\section{Future Directions and Concluding Remarks}

The present article has integrated various clinically relevant research lines and discussed developmental notions of identity structure and processes to increase our understanding of how certain psychopathological behaviors may emerge and develop. In doing so, we focused mainly on research investigating behaviors that "target the body" - ED symptomatology and NSSI. Hence, although these initial research efforts certainly have their merit as they explicitly direct our attention to how these behaviors may be linked to identity, more systematic and in-depth research is urgently needed to place these findings into a broader perspective. Partially inspired by authors such as Klimstra and Denissen (2017) and Kaufman et al. (2014), we briefly discuss some of these challenges for future research programs.

First, as previously discussed, we reiterate the need to investigate whether similar pathways as outlined in the present article are at work when relating non-body related pathological behaviors to identity problems. Only when such research has been conducted systematically across different pathological behaviors can the trans-diagnostic value of identity be corroborated and/or can the exact role of identity mechanisms can be pinpointed in specific pathologies. An important challenge in this respect is to assess the incremental predictive validity of identity over and above other, well-established predictors. In doing so, both broad trans-diagnostic vulnerabilities for psychopathology (e.g., impulsivity, negative affectivity, neurobiological markers) and specific risky behavioral processes (e.g., dysregulated behaviors, 
negative self-evaluations) should be incorporated in future studies to explore how identity may play into psychopathology (Kaufman \& Crowell, 2018). In the ideal scenario, such research would adopt a lifespan perspective, as identity may function as a more proximal intervening mechanism (e.g., mediator) linking psychopathology to well-established factors such as childhood trauma (Kaufman et al., 2014; Klimstra \& Denissen, 2017).

Second, the present article focused primarily on research assessing individuals' identity structure and the behavioral processes of exploration and commitment, and does not attend to the content of the identity being developed. However, as highlighted above, if we want to understand the identity functions of pathological behavior, we should consider both identity process/structure and identity content (McLean, Syed, \& Shucard, 2016). The importance of identity content for the understanding of psychopathology led Klimstra and Denissen (2017) to propose a multidimensional theoretical framework in which, as noted above, one's identity content (e.g., the internalization of the body perfect ideal; Vartanian, 2009; Wängqvist \& Frisén, 2013) and psychopathological symptoms may become increasingly interwoven over time. A narrative identity approach may be especially suitable for examining how in a subjective process of meaning making identity content and psychopathology may become increasingly intermixed throughout personal development (Adler, Lodi-Smith, Philippe, \& Houle, 2016; McAdams \& McLean, 2013). With regard to body image, a recent qualitative study in young adults (Kling et al., 2018) investigated both positive and negative ways in which the body is salient in identity. Thematic analysis identified four main themes (identification with the body, body functionality in performing identity-relevant tasks, appearance and identity in social interactions, and identity-relevant bodily engagement) and provides information on the interindividual differences with respect to this body-identity integration. Further, Dings (2019) has elaborated on the dynamic interplay between narrative identity and embodiment, focusing on the underlying mechanisms that could be at work. In his paper, he describes the debate on this 
matter that points to a potential bidirectional relation linking both constructs. More specifically, embodiment can be regarded a prerequisite for narrative self-understanding, while changes in the narrative also seem to affect one's experience of the body.

Third, closely aligned with this notion of identity content, we briefly discussed that behaviors such as NSSI may also have important social identity functions in that they may play into a sense of group belonging, as individuals may affiliate with others displaying similar behaviors ("we are self-injurers"). This phenomenon can be observed, for instance, in school or other peer contexts (Breen et al., 2013; Giletta, Burk, Scholte, Engels, \& Prinstein, 2013; Muehlenkamp, Brausch, Quigley, \& Whitlock, 2013) as well as in online communities, such as the so-called pro-ana and pro-mia websites. However, the recent research lines discussed in the present article mainly focused on ego-identity and personal identity, somewhat at the expense of social identity mechanisms. Further, personal and social identity mechanisms intersect in that certain group memberships may predominantly shape one's personal sense of identity as they become their main defining identity asset, somewhat at the expense of other identity domains (Albarello, Crocetti, \& Rubini, 2018; Erikson, 1968). In other words, one's identity may become engulfed with these specific contents related to pathological behavior (see Klimstra \& Denissen, 2017 for a more extended discussion). In sum, to fully understand how identity and psychopathology are related, research targeting identity at the personal, relational, and social levels is urgently needed (cf. Schwartz, Luyckx, et al., 2011).

Fourth, most identity research originating from the West is based on the notion that a consistent sense of self (i.e., identity synthesis from an Eriksonian perspective) plays into general wellbeing and positive mental health (Erikson, 1950, 1968). However, cultural psychologists highlight that the need for such a consistent self is more likely a feature of Western cultures than of Eastern cultures (Church et al., 2008; Markus \& Kitayama, 1991; Suh, 2002). Given the collectivistic nature of most of the Eastern cultures, a flexible sense of self - 
which changes and molds itself in accordance to the needs of different interpersonal relations can aid in maintaining social harmony. Consequently, self-consistency may not be as an important goal in Eastern cultures as it is in Western cultures (Heine, 2001; Heine \& Lehman, 1999; Suh, 2002). Empirical research has indeed shown that a lack of a consistent sense of self in Eastern samples often has a weaker link with poor psychological outcomes as compared to Western samples (Church et al., 2008; Kanagawa, Cross, \& Markus, 2001; Markus \& Kitayama, 1991; Suh, 2002). Some preliminary NSSI research does seem to support this observation. Gandhi, Luyckx, Adhikari, et al. (2018) found disturbances in identity formation (low identity synthesis and high identity confusion) to be stronger predictors of NSSI in Belgian youth as compared to Indian youth. In summary, existing research and theory suggest that crosscultural replication is necessary to determine whether similar mechanisms linking identity and (body-related) pathological behaviors can be found in different cultures.

Finally, the existing body of research has investigated identity formation primarily from a between-person perspective, comparing individuals' scores relative to the scores of other individuals in the sample. Although this approach provides valuable information, it fails to examine how certain (identity) processes and mechanisms fluctuate over time within the individual. As identity formation is regarded a highly idiosyncratic developmental process, a within-person modeling approach provides important information as well (Becht, Nelemans, Branje, Vollebergh, Koot, \& Meeus, 2017; Lichtwarck-Aschoff, van Geert, Bosma, \& Kunnen, 2008). To illustrate, a recent study by Bogaerts et al. (2018) examined the directionality of effects linking identity structure and processes in adolescence by focusing on both betweenand within-person associations. Combining these two perspectives, the study was able to identify (1) which individuals experienced substantial identity difficulties compared to their peers (i.e., at the between-person level); and (2) which mechanisms were at work in the intrapersonal development of such identity issues (i.e., at the within-person level). Hence, 
applying both between-person and within-person analyses are important in identity research, as both perspectives can uncover important parts of the puzzle.

In sum, in line with recent work (e.g., Klimstra \& Denissen, 2017), it is our hope that the present article may help in bringing neo-Eriksonian identity research closer again to where it originated from, that is, the clinic, such that the clinical and practical potential and utility of the identity construct may reach its full potential in the years to come. 


\section{References}

Adler, J. M., Lodi-Smith, J., Philippe, F. L., \& Houle, I. (2016). The incremental validity of narrative identity in predicting well-being: A review of the field and recommendations for the future. Personality and Social Psychology Review, 20, 142-175. doi:10.1177/1088868315585068

Albarello, F., Crocetti, E., \& Rubini, M. (2018). I and us: A longitudinal study on the interplay of personal and social identity in adolescence. Journal of Youth and Adolescence, 47, 689-702. doi:10.1007/s10964-017-0791-4

American Psychiatric Association. (2000). Diagnostic and statistical manual of mental disorders (4th ed., text revision.). Washington, D.C.: Author.

American Psychiatric Association. (2013). Diagnostic and statistical manual of mental disorders (5th ed., text revision.). Washington, D.C.: Author.

Archer, S. L., \& Waterman, A. S. (1990). Varieties of identity diffusions and foreclosures: An exploration of subcategories of the identity statuses. Journal of Adolescent Research, 5, 96-111. doi:10.1177/074355489051009

Arnett, J. J. (2000). Emerging adulthood. American Psychologist, 55, 469-480. doi:10.1037/0003-066X.55.5.469

Asch, S. S. (1971). Wrist scratching as a symptom of anhedonia: A predepressive state. The Psychoanalytic Quarterly, 40, 603-617. doi:10.1080/21674086.1971.11926577

Auslander, B. A., \& Dunham, R. M. (1996). Bulimia and the diffusion status of ego identity formation: Similarities of the empirical descriptors of self and parent. Journal of Adolescence, 19, 333-338. doi:10.1006/jado.1996.0031

Becht, A. I., Nelemans, S. A., Branje, S. J., Vollebergh, W. A., Koot, H. M., \& Meeus, W. H. (2017). Identity uncertainty and commitment making across adolescence: Five-year 
within-person associations using daily identity reports. Developmental Psychology, 53, 2103-2112. doi:10.1037/dev0000374

Berman, S. L., Montgomery, M. J., \& Kurtines, W. M. (2004). The development and validation of a measure of identity distress. Identity: An International Journal of Theory and Research, 4, 1-8. doi:10.1207/S1532706XID0401_1

Berzonsky, M. D. (2004). Identity processing style, self-construction, and personal epistemic assumptions: A social-cognitive perspective. European Journal of Developmental Psychology, 1, 303-315. doi:10.1080/17405620444000120

Bogaerts, A., Claes, L., Schwartz, S. J., Becht, A. I., Verschueren, M., Gandhi, A., \& Luyckx, K. (2018). Identity structure and processes in adolescence: Examining the directionality of between-and within-person associations. Journal of Youth and Adolescence, 1-17. doi:10.1007/s10964-018-0931-5

Breen, A. V., Lewis, S. P., \& Sutherland, O. (2013). Brief report: Non-suicidal self-injury in the context of self and identity development. Journal of Adult Development, 20, 57-62. doi:10.1007/s10804-013-9156-8

Bresin, K., \& Schoenleber, M. (2015). Gender differences in the prevalence of nonsuicidal selfinjury: A meta-analysis. Clinical Psychology Review, 38, 55-64. doi:10.1016/j.cpr.2015.02.009

Briere, J., \& Gil, E. (1998). Self-mutilation in clinical and general population samples: Prevalence, correlates, and functions. American Journal of Orthopsychiatry, 68, 609620. doi:10.1037/h0080369

Bruch, H. (1981). Developmental considerations of anorexia nervosa and obesity. Canadian Journal of Psychiatry, 26, 212-217.

Bruch, H. (1982). Anorexia nervosa: Therapy and theory. American Journal of Psychiatry, 139, 1531-1538. 
Campbell, J. D. (1990). Self-esteem and clarity of the self-concept. Journal of Personality and Social Psychology, 59, 538-549.

Campbell, J. D., Trapnell, P. D., Heine, S. J., Katz, I. M., Lavallee, L. F., \& Lehman, D. R. (1996). Self-concept clarity: Measurement, personality correlates, and cultural boundaries. Journal of Personality and Social Psychology, 70, 141-156. doi:10.1037/0022-3514.70.1.141

Cash, T. F., \& Deagle, E. A. (1997). The nature and extent of body-image disturbances in anorexia nervosa and bulimia nervosa: A meta-analysis. International Journal of Eating Disorders, $\quad 22, \quad 107-126$. doi:10.1002/(SICI)1098-108X(199709)22:2<107::AIDEAT1>3.0.CO;2-J

Casper, R. C. (1983). Some provisional ideas concerning psychological structure in anorexia nervosa and bulimia. In P. L. Darby, P. E. Garfinkel, D. M. Garner, \& D. V. Coscina (Eds.), Anorexia nervosa: Recent developments in research (pp. 378-392). New York, NY: Alan R. Liss.

Church, A. T., Anderson-Harumi, C. A., Del Prado, A. M., Curtis, G. J., Tanaka-Matsumi, J., Valdez Medina, J. L., . . Katigbak, M. S. (2008). Culture, cross-role consistency, and adjustment: Testing trait and cultural psychology perspectives. Journal of Personality and Social Psychology, 95, 739-755. doi:10.1037/0022-3514.95.3.739

Claes, L., Klonsky, E. D., Muehlenkamp, J., Kuppens, P., \& Vandereycken, W. (2010). The affect-regulation function of nonsuicidal self-injury in eating-disordered patients: Which affect states are regulated? Comprehensive Psychiatry, 51, 386-392. doi:10.1016/j.comppsych.2009.09.001

Claes, L., Luyckx, K., \& Bijttebier, P. (2014). Non-suicidal self-injury in adolescents: Prevalence and associations with identity formation above and beyond depression. Personality and Individual Differences, 61, 101-104. doi:10.1016/j.paid.2013.12.019 
Claes, L., Luyckx, K., Bijttebier, P., Turner, B., Ghandi, A., Smets, J., . . Goris, Y. (2015). Non-suicidal self-injury in patients with eating disorder: Associations with identity formation above and beyond anxiety and depression. European Eating Disorders Review, 23, 119-125. doi:10.1002/erv.2341

Claes, L., Luyckx, K., Vogel, B., Verschueren, M., \& Müller, A. (2018). Identity processes and clusters in individuals with and without pathological buying. Psychiatry Research, 267, 467-472. doi:10.1016/j.psychres.2018.06.003

Claes, L., \& Muehlenkamp, J. J. (2014). Non-suicidal self-injury in eating disorders: Advancements in etiology and treatment. Berlin, Germany: Springer.

Claes, L., Müller, A., \& Luyckx, K. (2016). Compulsive buying and hoarding as identity substitutes: The role of materialistic value endorsement and depression. Comprehensive Psychiatry, 68, 65-71. doi:10.1016/j.comppsych.2016.04.005

Claes, L., Van den Eynde, F., Guillaume, S., Vogels, C., \& Audenaert, K. (2012). Executive functioning in borderline personality disorder with and without self-harming behaviors. In A. M. Columbus (Ed.), Advances in Psychology Research (Vol. 89, pp. 177-189). New York, NY: Nova Science Publishers.

Corning, A. F., \& Heibel, H. D. (2016). Re-thinking eating disorder prevention: The case for prioritizing the promotion of healthy identity development. Eating Disorders, 24, 106113. doi:10.1080/10640266.2015.1034057

Côté, J. E., \& Levine, C. (1987). A formulation of Erikson's theory of ego identity formation. Developmental Review, 7, 273-325. doi:10.1016/0273-2297(87)90015-3

Côté, J. E., \& Levine, C. G. (2002). Identity, formation, agency, and culture: A social psychological synthesis. Mahwah, NJ: Lawrence Erlbaum Associates. 
Côté, J. E., \& Schwartz, S. J. (2002). Comparing psychological and sociological approaches to identity: Identity status, identity capital, and the individualization process. Journal of Adolescence, 25, 571-586. doi:10.1006/jado.2002.0511

Crocetti, E., Luyckx, K., Scrignaro, M., \& Sica, L. S. (2011). Identity formation in Italian emerging adults: A cluster-analytic approach and associations with psychosocial functioning. European Journal of Developmental Psychology, 8, 558-572. doi:10.1080/17405629.2011.576858

Croll, J., Neumark-Sztainer, D., Story, M., \& Ireland, M. (2002). Prevalence and risk and protective factors related to disordered eating behaviors among adolescents: Relationship to gender and ethnicity. Journal of Adolescent Health, 31, 166-175. doi:10.1016/S1054-139X(02)00368-3

Cucchi, A., Ryan, D., Konstantakopoulos, G., Stroumpa, S., Kaçar, A., Renshaw, S., . . . Kravariti, E. (2016). Lifetime prevalence of non-suicidal self-injury in patients with eating disorders: A systematic review and meta-analysis. Psychological Medicine, 46, 1345-1358. doi:10.1017/S0033291716000027

Cushman, P. (1990). Why the self is empty: Toward a historically situated psychology. American Psychologist, 45, 599-611. doi:10.1037/0003-066X.45.5.599

Dings, R. (2019). The dynamic and recursive interplay of embodiment and narrative identity. Philosophical Psychology, 32, 186-210. doi:10.1080/09515089.2018.1548698

Dittmar, H., Long, K., \& Bond, R. (2007). When a better self is only a button click away: Associations between materialistic values, emotional and identity-related buying motives, and compulsive buying tendency online. Journal of Social and Clinical Psychology, 26, 334-361. doi:10.1521/jscp.2007.26.3.334

Elkind, D., \& Bowen, R. (1979). Imaginary audience behavior in children and adolescents. Developmental Psychology, 15, 38. doi:10.1037/0012-1649.15.1.38 
Erikson, E. H. (1950). Childhood and society. New York, NY: W. W. Norton.

Erikson, E. H. (1968). Identity: Youth and crisis. New York, NY: Norton.

Fairburn, C. G., Cooper, Z., \& Shafran, R. (2003). Cognitive behaviour therapy for eating disorders: A "transdiagnostic" theory and treatment. Behaviour Research and Therapy, 41, 509-528. doi:10.1016/S0005-7967(02)00088-8

Frost, R. O., Kyrios, M., McCarthy, K. D., \& Matthews, Y. (2007). Self-ambivalence and attachment to possessions. Journal of Cognitive Psychotherapy, 21, 232-242. doi:10.1891/088983907781494582

Gandhi, A., Luyckx, K., Adhikari, A., Parmar, D., De Sousa, A., Shah, N., . . Claes, L. (2018). Non-suicidal self-injury and its association with identity formation in India and Belgium: A cross-cultural case-control study. Manuscript submitted for publication.

Gandhi, A., Luyckx, K., Baetens, I., Kiekens, G., Sleuwaegen, E., Berens, A., . . Claes, L. (2018). Age of onset of non-suicidal self-injury in Dutch-speaking adolescents and emerging adults: An event history analysis of pooled data. Comprehensive Psychiatry, 80, 170-178. doi:10.1016/j.comppsych.2017.10.007

Gandhi, A., Luyckx, K., Goossens, L., Maitra, S., \& Claes, L. (2016). Sociotropy, autonomy, and non-suicidal self-injury: The mediating role of identity confusion. Personality and Individual Differences, 99, 272-277. doi:10.1016/j.paid.2016.05.040

Gandhi, A., Luyckx, K., Maitra, S., \& Claes, L. (2015). Non-suicidal self-injury and identity distress in Flemish adolescents: Exploring gender differences and mediational pathways. Personality and Individual Differences, 82, 215-220. doi:10.1016/j.paid.2015.03.031

Gandhi, A., Luyckx, K., Maitra, S., Kiekens, G., Verschueren, M., \& Claes, L. (2017). Directionality of effects between non-suicidal self-injury and identity formation: A 
prospective study in adolescents. Personality and Individual Differences, 109, 124-129. doi:10.1016/j.paid.2017.01.003

Giletta, M., Burk, W. J., Scholte, R. H., Engels, R. C., \& Prinstein, M. J. (2013). Direct and indirect peer socialization of adolescent nonsuicidal self-injury. Journal of Research on Adolescence 23, 450-463. doi:10.1111/jora.12036

Harter, S. (1999). The construction of the self: A developmental perspective. New York, NY: Guilford Press.

Heatherton, T. F., \& Baumeister, R. F. (1991). Binge eating as escape from self-awareness. Psychological Bulletin, 110, 86-108. doi:10.1037/0033-2909.110.1.86

Heine, S. J. (2001). Self as cultural product: An examination of East Asian and North American selves. Journal of Personality, 69, 881-905. doi:10.1111/1467-6494.696168

Heine, S. J., \& Lehman, D. R. (1999). Culture, self-discrepancies, and self-satisfaction. Personality and Social Psychology Bulletin, 25, 915-925. doi:10.1177/01461672992511001

Herman, C. P., \& Polivy, J. (1988). Excess and restraint in bulimia. In K. Pirke, W. Vandereycken, \& D. Ploog (Eds.), The psychobiology of bulimia (pp. 33-41). NY, New York: Springer-Verlag.

Kanagawa, C., Cross, S. E., \& Markus, H. R. (2001). "Who am I?” The cultural psychology of the conceptual self. Personality and Social Psychology Bulletin, 27, 90-103. doi:10.1177/0146167201271008

Kaufman, E. A., \& Crowell, S. E. (2018). Biological and behavioral mechanisms of identity pathology development: An integrative review. Review of General Psychology, 22, 245263. doi:10.1037/gpr0000138 
Kaufman, E. A., Montgomery, M., \& Crowell, S. (2014). Identity-related dysfunction: Integrating clinical and developmental perspectives. Identity, 14, 297-311. doi:10.1080/15283488.2014.944699

Kernberg, O. (1984). Severe personality disorders. New Haven, CT: Yale University Press.

Kerr, P. L., Muehlenkamp, J. J., \& Turner, J. M. (2010). Nonsuicidal self-injury: A review of current research for family medicine and primary care physicians. The Journal of the American Board of Family Medicine, 23, 240-259. doi:10.3122/jabfm.2010.02.090110

Klimstra, T. A., \& Denissen, J. J. (2017). A theoretical framework for the associations between identity and psychopathology. Developmental Psychology, 53, 2052-2065. doi:10.1037/dev0000356

Kling, J., Wängqvist, M., \& Frisén, A. (2018). “This body is me” Discovering the ways in which the body is salient in people's identities. Body image, 24, 102-110. doi:10.1016/j.bodyim.2017.12.009

Kroger, J. (2000). Ego identity status research in the new millennium. International Journal of Behavioral Development, 24, 145-148. doi:10.1080/016502500383250

Lewinsohn, P. M., Seeley, J. R., Moerk, K. C., \& Striegel-Moore, R. H. (2002). Gender differences in eating disorder symptoms in young adults. International Journal of Eating Disorders, 32, 426-440. doi:10.1002/eat.10103

Lichtwarck-Aschoff, A., van Geert, P., Bosma, H., \& Kunnen, S. (2008). Time and identity: A framework for research and theory formation. Developmental Review, 28, 370-400. doi:10.1016/j.dr.2008.04.001

Luyckx, K., Gandhi, A., Bijttebier, P., \& Claes, L. (2015a). Non-suicidal self-injury in female adolescents and psychiatric patients: A replication and extension of the role of identity formation. Personality and Individual Differences, $77, \quad 91-96$. doi:10.1016/j.paid.2014.12.057 
Luyckx, K., Gandhi, A., Bijttebier, P., \& Claes, L. (2015b). Non-suicidal self-injury in high school students: Associations with identity processes and statuses. Journal of Adolescence, 41, 76-85. doi:10.1016/j.adolescence.2015.03.003

Luyckx, K., Goossens, L., \& Soenens, B. (2006). A developmental contextual perspective on identity construction in emerging adulthood: Change dynamics in commitment formation and commitment evaluation. Developmental Psychology, 42, 366-380. doi:10.1037/0012-1649.42.2.366

Luyckx, K., Klimstra, T. A., Duriez, B., Van Petegem, S., \& Beyers, W. (2013). Personal identity processes from adolescence through the late 20s: Age trends, functionality, and depressive symptoms. Social Development, 22, 701-7021. doi:10.1111/sode.12027

Luyckx, K., Klimstra, T. A., Schwartz, S. J., \& Duriez, B. (2013). Personal identity in college and the work context: Developmental trajectories and psychosocial functioning. European Journal of Personality, 27, 222-237. doi:10.1002/per.1903

Luyckx, K., Schwartz, S. J., Berzonsky, M. D., Soenens, B., Vansteenkiste, M., Smits, I., \& Goossens, L. (2008). Capturing ruminative exploration: Extending the four-dimensional model of identity formation in late adolescence. Journal of Research in Personality, 42, 58-82. doi:10.1016/j.jrp.2007.04.004

Mannerström, R., Hautamäki, A., \& Leikas, S. (2017). Identity status among young adults: Validation of the Dimensions of Identity Development Scale (DIDS) in a Finnish sample. Nordic Psychology, 69, 195-213. doi:10.1080/19012276.2016.1245156

Marcia, J. (1966). Development and validation of ego-identity status. Journal of Personality and Social Psychology, 3, 551-558. doi:10.1037/h0023281

Marcia, J. (1989). Identity and intervention. Journal of Adolescence, 12, 401-410. 
Marcia, J. (2002). Identity and psychosocial development in adulthood. Identity: An International Journal of Theory and Research, 2, 7-28. doi:10.1207/S1532706XID0201_02

Marcia, J. (2006). Ego identity and personality disorders. Journal of personality disorders, 20, $577-596$.

Markus, H. R., \& Kitayama, S. (1991). Culture and the self: Implications for cognition, emotion, and motivation. Psychological Review, 98, 224-253. doi:10.1037/0033295X.98.2.224

McAdams, D. P., \& McLean, K. C. (2013). Narrative identity. Current Directions in Psychological Science, 22, 233-238. doi:10.1177/0963721413475622

McLean, K. C., Syed, M., \& Shucard, H. (2016). Bringing identity content to the fore: Links to identity development processes. Emerging Adulthood, 4, 356-364. doi:10.1177/2167696815626820

Micali, N., Hagberg, K. W., Petersen, I., \& Treasure, J. L. (2013). The incidence of eating disorders in the UK in 2000-2009: Findings from the General Practice Research Database. BMJ open, 3, 1-8. doi:10.1136/bmjopen-2013-002646

Micali, N., Ploubidis, G., De Stavola, B., Simonoff, E., \& Treasure, J. (2014). Frequency and patterns of eating disorder symptoms in early adolescence. Journal of Adolescent Health, 54, 574-581. doi:10.1016/j.jadohealth.2013.10.200

Muehlenkamp, J. J., Brausch, A., Quigley, K., \& Whitlock, J. (2013). Interpersonal features and functions of nonsuicidal self-injury. Suicide and Life-Threatening Behavior, 43, 6780. doi:10.1111/j.1943-278X.2012.00128.X

Muehlenkamp, J. J., \& Brausch, A. M. (2012). Body image as a mediator of non-suicidal selfinjury in adolescents. Journal of Adolescence, 35, 1-9. doi:10.1016/j.adolescence.2011.06.010Get 
Muehlenkamp, J. J., Claes, L., Havertape, L., \& Plener, P. L. (2012). International prevalence of adolescent non-suicidal self-injury and deliberate self-harm. Child and Adolescent Psychiatry and Mental Health, 6, Article ID 10. doi:10.1186/1753-2000-6-10

Muehlenkamp, J. J., Claes, L., Smits, D., Peat, C. M., \& Vandereycken, W. (2011). Nonsuicidal self-injury in eating disordered patients: A test of a conceptual model. Psychiatry Research, 188, 102-108. doi:10.1016/j.psychres.2010.12.023

Muehlenkamp, J. J., \& Saris-Baglama, R. N. (2002). Self-objectification and its psychological outcomes for college women. Psychology of Women Quarterly, 26, 371-379. doi:10.1111/1471-6402.t01-1-00076

Muehlenkamp, J. J., Swanson, J. D., \& Brausch, A. M. (2005). Self-objectification, risk taking, and self-harm in college women. Psychology of Women Quarterly, 29, 24-32. doi:10.1111/j.1471-6402.2005.00164.x

Mullins-Sweatt, S. N., Lengel, G. J., \& Grant, D. M. (2013). Non-suicidal self-injury: The contribution of general personality functioning. Personality and Mental Health, 7, 5668. doi:10.1002/pmh.1211

Nock, M. K. (2009). Understanding nonsuicidal self-injury. Origins, assessment and treatment. Washington, DC: American Psychological Association.

Nock, M. K., \& Favazza, A. R. (2009). Non-suicidal self-injury: Origins, assessment, and treatment. Washington, DC: American Psychological Association.

Nock, M. K., Joiner, T. E., Gordon, K. H., Lloyd-Richardson, E., \& Prinstein, M. J. (2006). Non-suicidal self-injury among adolescents: Diagnostic correlates and relation to suicide attempts. Psychiatry Research, 144, 65-72. doi:10.1016/j.psychres.2006.05.010

Orbach, I. (1996). The role of the body experience in self-destruction. Clinical Child Psychology and Psychiatry, 1, 607-619. doi:10.1177/1359104596014012 
Orbach, I., \& Mikulincer, M. (1998). The Body Investment Scale: Construction and validation of a body experience scale. Psychological Assessment, 10, 415-425. doi:10.1037/10403590.10.4.415

Pasupathi, M. (2014). Identity: Commentary: Identity development: Dialogue between normative and pathological developmental approaches. Journal of personality disorders, 28, 113-120. doi:10.1521/pedi.2014.28.1.113

Plener, P. L., Schumacher, T. S., Munz, L. M., \& Groschwitz, R. C. (2015). The longitudinal course of non-suicidal self-injury and deliberate self-harm: A systematic review of the literature. Borderline Personality Disorder and Emotion Dysregulation, 2, 2. doi:10.1186/s40479-014-0024-3

Podvoll, E. M. (1969). Self-mutilation within a hospital setting: A study of identity and social compliance. British Journal of Medical Psychology, 42, 213-221. doi:10.1111/j.20448341.1969.tb02073.x

Polivy, J., Herman, C. P., \& McFarlane, T. (1994). Effects of anxiety on eating: Does palatability moderate distress-induced overeating in dieters? Journal of Abnormal Psychology, 103, 505-510. doi:10.1037/0021-843X.103.3.505

Polivy, J., \& Herman, P. (2007). Is the body the self? Women and body image. Collegium Antropologicum, 31, 63-67.

Quick, V. M., \& Byrd-Bredbenner, C. (2013). Disturbed eating behaviours and associated psychographic characteristics of college students. Journal of Human Nutrition and Dietetics, 26, 53-63. doi:10.1111/jhn.12060

Reeves, R. A., Baker, G. A., \& Truluck, C. S. (2012). Celebrity worship, materialism, compulsive buying, and the empty self. Psychology \& Marketing, 29, 674-679. doi:10.1002/mar.20553 
Schmeck, K., Schlüter-Müller, S., Foelsch, P. A., \& Doering, S. (2013). The role of identity in the DSM-5 classification of personality disorders. Child and Adolescent Psychiatry and Mental Health, 7, 27. doi:10.1186/1753-2000-7-27

Schupak-Neuberg, E., \& Nemeroff, C. J. (1993). Disturbances in identity and self-regulation in bulimia nervosa: Implications for a metaphorical perspective of "body as self". International Journal of Eating Disorders, 13, 335-347. doi:10.1002/1098108X(199305)13:4<335::AID-EAT2260130402>3.0.CO;2-M

Schwartz, S. J., Beyers, W., Luyckx, K., Soenens, B., Zamboanga, B., Forthun, L., . . . Waterman, A. (2011). Examining the light and dark sides of emerging adults' identity: A study of identity status differences in positive and negative psychosocial functioning. Journal of Youth and Adolescence, 40, 839-859. doi:10.1007/s10964-010-9606-6

Schwartz, S. J., Côté, J. E., \& Arnett, J. J. (2005). Identity and agency in emerging adulthood: Two developmental routes in the individualization process. Youth \& Society, 37, 201229. doi:10.1177/0044118X05275965

Schwartz, S. J., Luyckx, K., \& Vignoles, V. L. (2011). Handbook of identity theory and research. New York, NY: Springer.

Sparks, M. D. (1993). The relationship between eating disorders and ego identity development. Unpublished doctoral dissertation. Utah State University. Logan, Utah.

Stanghellini, G., Castellini, G., Brogna, P., Faravelli, C., \& Ricca, V. (2012). Identity and Eating Disorders (IDEA): A questionnaire evaluating identity and embodiment in eating disorder patients. Psychopathology, 45, 147-158. doi:10.1159/000330258

Stuurgroep Eetstoornissen Nederland. (1998). Eindrapport: Leidschendam: SEN.

Suh, E. M. (2002). Culture, identity consistency, and subjective well-being. Journal of Personality and Social Psychology, 83, 1378-1391. doi:10.1037/0022-3514.83.6.1378 
Suisman, J. L., Thompson, J. K., Keel, P. K., Burt, S. A., Neale, M., Boker, S., . . Klump, K. L. (2014). Genetic and environmental influences on thin-ideal internalization across puberty and preadolescent, adolescent, and young adult development. International Journal of Eating Disorders, 47, 773-783. doi:10.1002/eat.22321

Svirko, E., \& Hawton, K. (2007). Self-injurious behavior and eating disorders: The extent and nature of the association. Suicide and Life-Threatening Behavior, 37, 409-421. doi:10.1521/suli.2007.37.4.409

Swannell, S. V., Martin, G. E., Page, A., Hasking, P., \& St John, N. J. (2014). Prevalence of nonsuicidal self-injury in nonclinical samples: Systematic review, meta-analysis and meta-regression. Suicide and Life-Threatening Behavior, 44, 273-303. doi:10.1111/sltb.12070

Swanson, S. A., Crow, S. J., Le Grange, D., Swendsen, J., \& Merikangas, K. R. (2011). Prevalence and correlates of eating disorders in adolescents: Results from the national comorbidity survey replication adolescent supplement. Archives of General Psychiatry, 68, 714-723. doi:10.1001/archgenpsychiatry.2011.22

Vandereycken, W., \& Noordenbos, G. (Eds.). (2008). Handboek eetstoornissen. Utrecht, the Netherlands: De Tijdstroom.

Vartanian, L. R. (2009). When the body defines the self: Self-concept clarity, internalization, and body image. Journal of Social and Clinical Psychology, 28, 94-126. doi:10.1521/jscp.2009.28.1.94

Vartanian, L. R., Hayward, L. E., Smyth, J. M., Paxton, S. J., \& Touyz, S. W. (2018). Risk and resiliency factors related to body dissatisfaction and disordered eating: The identity disruption model. International Journal of Eating Disorders, 51, 322-330. doi:10.1002/eat.22835 
Verschueren, M., Claes, L., Bogaerts, A., Palmeroni, N., Gandhi, A., Moons, P., \& Luyckx, K. (2018). Eating disorder symptomatology and identity formation in adolescence: A cross-lagged longitudinal approach. Frontiers in Psychology, 9. doi:10.3389/fpsyg.2018.00816

Verschueren, M., Luyckx, K., Kaufman, E. A., Vansteenkiste, M., Moons, P., Sleuwaegen, E., .. Claes, L. (2017). Identity processes and statuses in patients with and without eating disorders. European Eating Disorders Review, 25, 26-35. doi:10.1002/erv.2487

Verstuyf, J., Van Petegem, S., Vansteenkiste, M., Soenens, B., \& Boone, L. (2014). The body perfect ideal and eating regulation goals: Investigating the role of adolescents' identity styles. Journal of Youth and Adolescence, 43, 284-297. doi:10.1007/s10964-013-9949-

$\mathrm{X}$

Wängqvist, M., \& Frisén, A. (2013). Swedish 18-year-olds' identity formation: Associations with feelings about appearance and internalization of body ideals. Journal of Adolescence, 36, 485-493. doi:10.1016/j.adolescence.2013.02.002

Waterman, A. (2007). Doing well: The relationship of identity status to three conceptions of well-being. Identity, 7, 289-307. doi:10.1080/15283480701600769

Westen, D., Betan, E., \& DeFife, J. A. (2011). Identity disturbance in adolescence: Associations with borderline personality disorder. Development and Psychopathology, 23, 305-313. doi:10.1017/S0954579410000817

Wheeler, H. A., Adams, G. R., \& Keating, L. (2001). Binge eating as a means for evading identity issues: The association between an avoidance identity style and bulimic behavior. Identity, 1, 161-178. doi:10.1207/S1532706XID0102_04

Wicklund, R. A., \& Gollwitzer, P. M. (1982). Symbolic self completion. Hillsdale, NJ: Erlbaum. Wiley, R. E., \& Berman, S. L. (2013). Adolescent identity development and distress in a clinical sample. Journal of Clinical Psychology, 69, 1299-1304. doi:10.1002/jclp.22004 


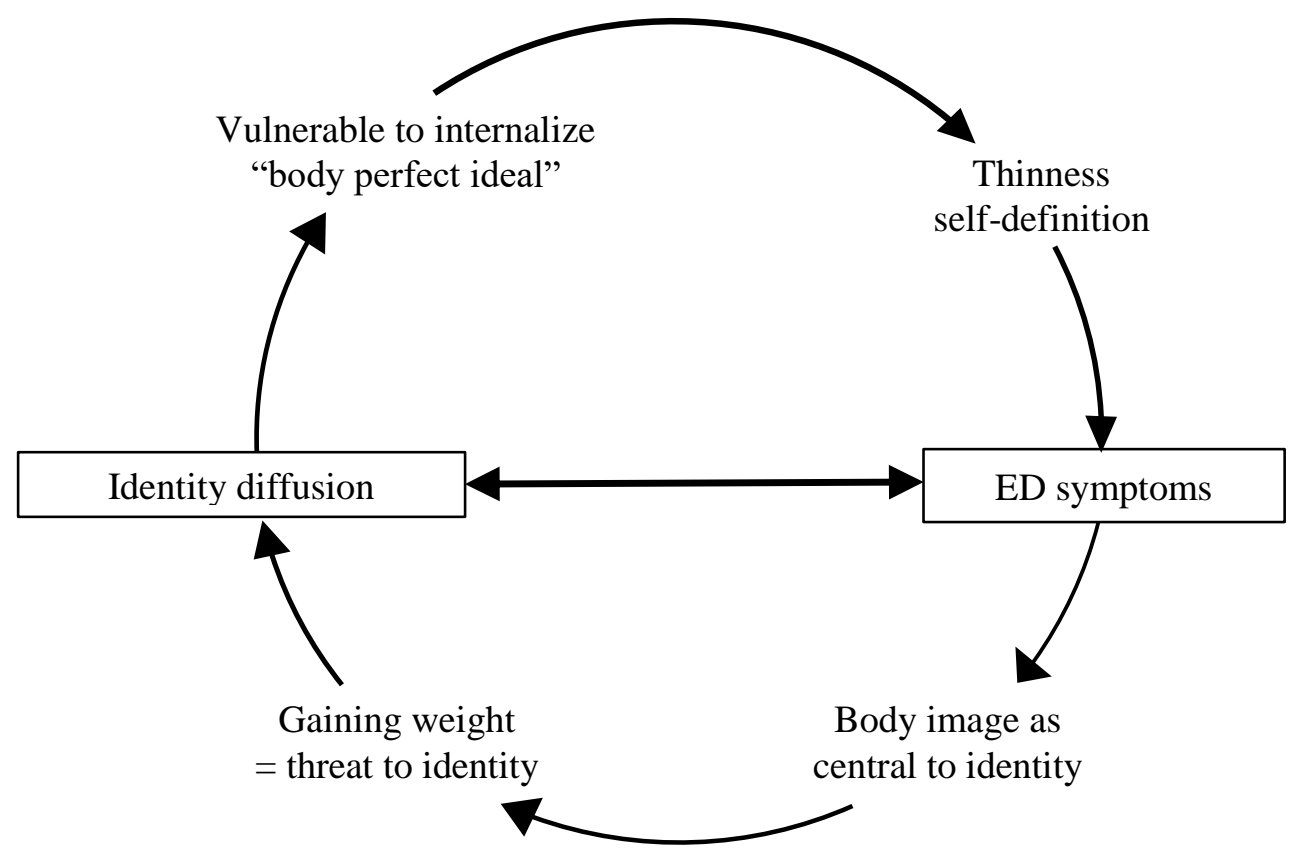

Figure 1. The identity - ED interplay

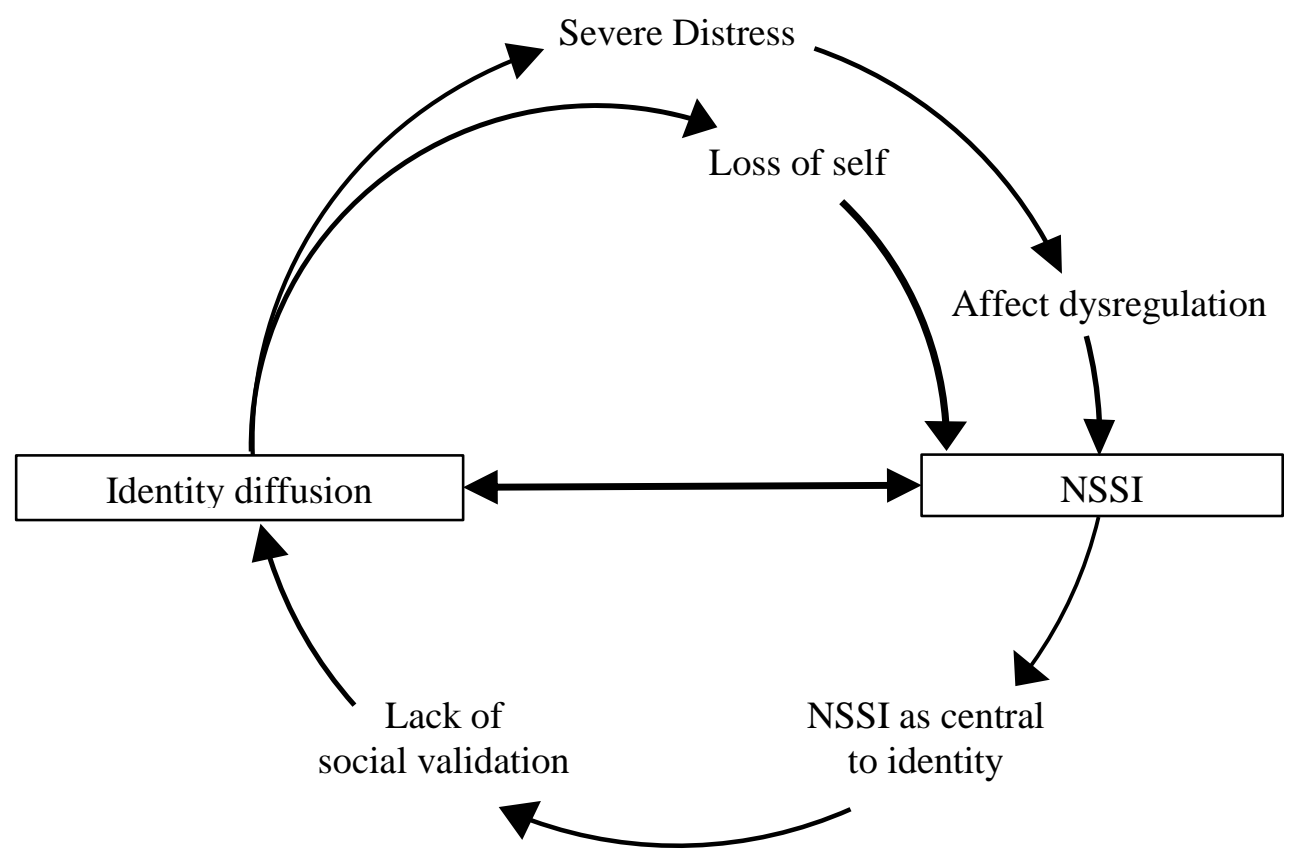

Figure 2. The identity - NSSI interplay 